\title{
基于位移残差的数据分发管理区域匹配及传输 算法
}

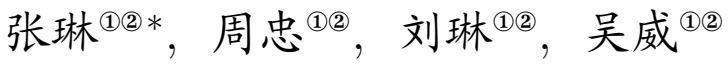 \\ (1) 北京航空航天大学虚拟现实技术与系统国家重点实验室, 北京 100191 \\ (2) 北京航空航天大学计算机学院, 北京 100191 \\ * 通信作者. E-mail: zhanglin@vrlab.buaa.edu.cn
}

收稿日期: 2011-04-06; 接受日期: 2011-05-30

国家重点基础研究发展计划（批准号：2009CB320805)、国家自然科学基金 (批准号：61073070)、2008 中国下一代互联网应用 示范子项目 (批准号: CNGI2008-123) 和中央高校基本科研业务费专项资金资助项目

\begin{abstract}
摘要 高层体系结构 (high level architecture, HLA) 是建模与仿真的国际标准, 它规定了数据分发管 理服务 (data distribution management, DDM) 来减少不相关数据的发送与接收, 从而减少网络带宽占 用. 发布区域与订购区域之间匹配计算的效率是影响数据分发管理服务质量的重要因素. 区域信息 的传输同样非常重要，盟员间通过区域信息的传输来保持区域的正确性和一致性，但区域信息的传 输同样会给网络带来负载. 现有算法通常是在区域保真度和网络负载两者之间做折中处理，难以兼 顾。因此, 本文充分考虑了变化区域的匹配效率以及区域传输的带宽占用问题,做出以下贡献: 1) 定 义了区域变化与区域匹配变化之间的映射关系, 采用该映射可以明显减少区域改变后所需要的匹配 计算次数, 从而提高了匹配效率; 2) 将区域更新信息划分为两种数据表达, 区域快照与区域残差. 通 过尽量传输区域残差来代替传输完整的区域信息可以有效减少区域信息传输所消耗的网络带宽. 因 此, 本文提出一种基于位移残差的 DDM 匹配与传输算法, 给出了算法的理论分析与测试结果, 并且 对算法在实际应用中的表现进行了测试对比, 给出了数据对比与结果分析. 实验结果表明, 基于位移 残差的 DDM 匹配与传输算法具有良好的匹配效率, 并且能够大大减少网络带宽占用, 尤其是在具有 大量不断变化区域的情况下.
\end{abstract}

关键词 分布式仿真 高层体系结构 数据分发管理 区域快照 区域残差

\section{1 引言}

大规模分布交互仿真中大量对象在同一个虚拟环境中运动与交互, 会持续产生巨大数量的报文. 数据的急剧增长限制了系统规模与效率. 兴趣管理技术被广泛应用于减少网络传输的数据量, 人们对 于兴趣管理技术的研究从 20 世纪 90 年代就已经开始了, 大多数兴趣管理技术都采用对象的空间属 性来表达兴趣. 高层体系结构 HLA 是建模与仿真的通用技术体系结构标准, 它规定了一组基于区域 的兴趣过滤服务, 叫做数据分发管理服务. 在 HLA 1.3 标准中, 更新区域、订购区域以及路由空间是 数据分发管理服务定义的 3 个重要概念 ${ }^{[1]}$. 在数据分发管理服务中, 数据生产者根据用户定义的发布 
区域发布数据属性, 数据消费者则根据用户定义的订购区域指定他们的数据需求, 然后由 RTI 根据数 据生产者与消费者之间发布与订购区域的匹配关系进行数据分发. 因此, 用来判断分布与订购区域是 否相交的区域匹配算法的计算效率, 成为数据分发管理服务的关键. 事实上, 由于区域匹配算法效率 和区域信息的传输效率在数据分发管理服务实现中都至关重要, 数据分发管理服务必须兼顾两者. 现 有的区域匹配算法根据匹配计算方法的不同可划分为以下 4 类: 基于区域的匹配算法 (region-based

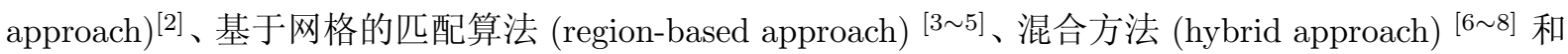
基于排序的匹配算法 (sort-based approach) ${ }^{[9 \sim 11]}$. 每种方法都有其各自的优缺点, 但它们都没有把区 域信息的传输考虑进去. 本文研究了基于排序匹配算法的扫描边界, 定义了区域变化与区域匹配变化 之间的映射关系, 并在此基础上提出基于位移残差的区域匹配算法, 该算法可有效减少区域改变后所 需要的匹配计算次数, 从而提高匹配效率. 在区域信息传输方面, 将区域更新信息划分为两种数据表 达, 区域快照与区域残差, 通过尽量传输区域残差来代替传输完整的区域信息, 达到减少区域信息传输 所消耗的网络带宽的目的.

\section{2 相关工作}

本节对现有 4 种主要的数据分发管理匹配算法进行描述, 并给出它们各自的优缺点.

基于区域的方法是一种穷举的方法, 它把每个更新区域和所有的订购区域一一比较来获得匹配信 息, 因此, 该方法计算得到的匹配信息是精确的。基于区域的方法是一种一对多的匹配方式,一个更新 区域要和所有订购区域进行匹配, 因此其计算复杂度为 $O\left(N^{2}\right)^{[5]}$. 并且, 当某一更新区域发生改变时, 需要重新计算该区域与所有订购区域的匹配关系, 因此不适用于区域变化频繁的情况.

为减少基于区域方法的计算复杂度, 人们提出一种基于网格的匹配方法, 该方法将路由空间划分 为一系列网格, 每个区域都被映射到这些网格上. 一个更新区域与一个订购区域被认为是相交的, 当 且仅当它们映射在至少一个共同网格上. 然而, 基于网格的匹配方法无法得到准确的区域相交信息, 因此接收方可能会收到不相关的数据报文 ${ }^{[12,13]}$.

基于区域的方法可以得到精确的匹配结果, 但是匹配计算效率低. 基于网格的方法具有较高的匹 配效率, 但是无法对区域进行精确匹配. 因此, Tan 等 ${ }^{[7]}$ 提出了一种混合方法, 这种方法结合了基于 区域匹配的方法和基于网格匹配的方法. 它先利用基于网格的方法将所有区域映射到网格单元当中, 然后在这些单元格内利用穷举法一一匹配所有的更新区域和订购区域. 这种方法结合了穷举法和网格 方法的优点, 与穷举法相比降低了计算的复杂度, 与网格方法相比它能够得到精确的匹配信息, 降低了 主机的数据处理量. 混合方法最主要的缺点是其性能取决于网格大小的选择.

Raczy 等 [11] 在穷举法基础上首次提出了一种基于排序的方法. 该方法将区域的端点插入到一个 排序的列表中, 通过遍历该有序列表计算出所有区域的匹配信息. 虽然该方法的复杂度仍然是 $O\left(N^{2}\right)$, 但是得益于位操作, 它有着不错的性能. 但是由于它 $O\left(N^{2}\right)$ 的存储复杂度, 它并不适合在大规模的分 布虚拟仿真中应用. 另外, 这种方法在有任何区域改变的情况下, 都必须对所有区域进行重新计算来 得到最新的匹配结果, 不适用于区域频繁变化的情况.

Pan 等 ${ }^{[10]}$ 提出了一种改进的基于排序的匹配算法, 进一步限定了匹配的范围, 使得在区域改变 时, 匹配可以缩小到一个较小的范围. 与原始的排序匹配算法不同的是, 该方法将所有订购区域的上 端点和下端点分别插入到两个排序的列表中, 对发布区域也是一样. 计算一个区域的匹配信息, 算法 分别在上端点列表和下端点列表的一定子范围中进行匹配判断. 由于缩小了计算范围, 该方法具有较 


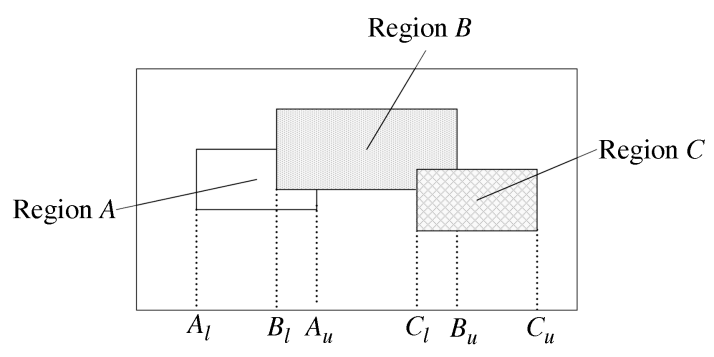

图 1 区域交叠实例

Figure 1 Example of region overlapping
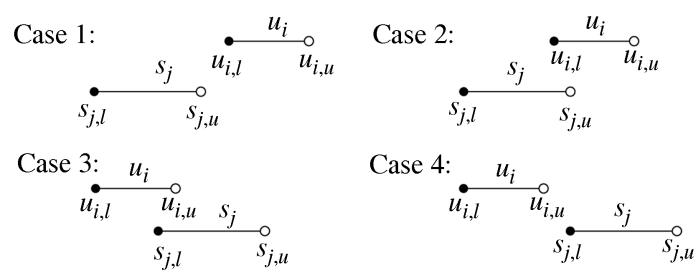

图 2 两个限域的不同交叠情况

Figure 2 Overlapping cases for two ranges

好的匹配效率. 但该方法仍存在明显缺陷, 当虚拟环境中大多数实体具有较小的兴趣区域, 而仅有少 数实体具有大的兴趣区域的时候, 采用最大兴趣区域限定匹配计算范围的方法仍然存在很多不必要的 计算.

本文提出的 DRBM 算法与现有基于排序的匹配算法具有明显不同之处. 首先, DRBM 算法进一 步缩短了匹配计算的范围, 并给出了相应的理论证明. 由于数据分发管理服务的效率主要由匹配算法 的效率决定, 缩小匹配计算的范围必然会提高匹配效率. 其次, 本文算法兼顾了匹配效率和网络传输, 通过区域残差的传输降低区域信息传输所占用的网络带宽, 而现有算法都没有考虑网络传输.

\section{3 区域匹配的端点特性}

为充分了解区域匹配的特性, 我们先从一个简单的例子开始入手. 图 1 给出了二维空间中的 3 个 不同区域, 区域 $A, B, C$. 从图中可以看出, 区域 $B$ 与区域 $A, C$ 是匹配的, 而区域 $A$ 和区域 $C$ 不匹配.

如图 1 所示, 如果把所有区域投影到公共的维上, 那么我们很容易通过对维上范围 (range) 的交 叠计算来得到区域在这一维上的匹配情况. 当且仅当两个区域在每一个公共维度上都相交时, 这两个 区域是匹配的. 因此, 对二维甚至多维路由空间中区域的匹配计算问题, 可以通过计算每一公共维度 的交叠信息来实现.

图 2 给出了同一维上区域的限域间所有可能的交叠关系. 如图 2 所示, 同一维上的限域 $u_{i}\left[u_{i, l}, u_{i, u}\right)$ 和 $s_{j}\left[s_{j, l}, s_{j, u}\right)$ 共有 4 种可能的位置关系, 可以看出两个限域相交叠的充分必要条件是同时满足不等 式 (1) 和 $(2)^{[10]}$.

$$
\begin{aligned}
& u_{i, u}>s_{j, l}, \\
& s_{j, u}>u_{i, l} .
\end{aligned}
$$

因此对于任意一个更新限域 $u_{i}$, 计算所有与之相交叠的订购限域的过程就是查找所有同时满足不 等式 (1) 和 (2) 的订购限域. 以更新限域交叠信息的计算为例, 如果每次都需要遍历所有的订购限域, 计算代价非常大, 尤其是在具有大量区域的大路径空间中. 本文首先研究如何减少交叠信息计算的匹 配次数.

在区域匹配关系上, 发布区域和订购区域都具有相同的特性. 因此, 为方便描述本文详细给出更 新限域 $u_{i}$ 与维中所有订购限域间交叠关系特性. 为更好地描述匹配问题, 我们定义了一些基本符号.

令 $\Phi$ 表示所有订购区域, $\Psi$ 表示所有更新区域. 令 $\phi^{d}, \psi^{d}$ 分别表示在维度 $d$ 上的所有订购限域 和发布限域. 给定更新区域 $U_{i}$, 令 $\phi_{i}$ 表示所有与 $U_{i}$ 相交叠的发布区域集合; 令 $\psi_{i}$ 表示与订购区域 $S_{i}$ 相交叠的所有发布区域的集合. 


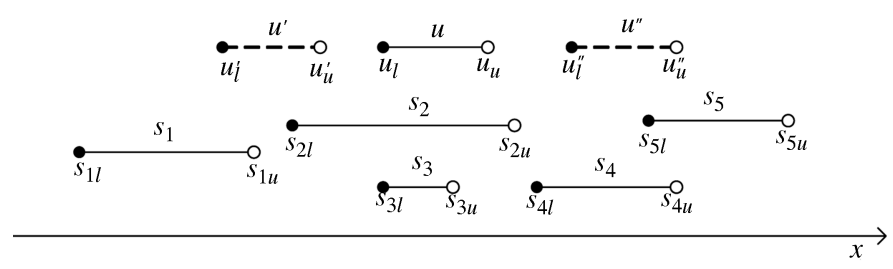

图 3 限域变化和匹配关系变化

Figure 3 Changes in overlap caused by modification of a range

令 $\phi_{i}^{d}$ 表示维度 $d$ 上与更新限域 $u_{i}$ 相交叠的所有订购限域的集合, 即 $\phi_{i}^{d}=\left\{s_{j} \mid s_{j}\right.$ 与 $u_{i}$ 相交叠, $\left.s_{j} \in \phi^{d}\right\}$. 同样地, 令 $\psi_{i}^{d}$ 表示维度 $d$ 上与订购限域 $s_{i}$ 相交叠的所有发布限域的集合, 即 $\psi_{i}^{d}=\left\{u_{j} \mid u_{j}\right.$ 与 $s_{i}$ 相交叠, $\left.u_{j} \in \psi^{d}\right\}$.

定义 1 最大订购限域长度 (maxSRS, maximum subscription range size). 对于给定维度 $d$, $\operatorname{maxSRS}$ 表示订购限域集合 $\phi^{d}$ 中最大的限域长度.

定义 2 最大发布限域长度 (maxURS, maximum update range size). 对于给定维度 $d$, maxURS 表示发布限域集合 $\psi^{d}$ 中最大的限域长度.

定理 1 对任意 $s_{j} \in \phi_{i}^{d}$, 有 $u_{i, l}-\operatorname{maxSRS}<s_{j, l}<u_{i, u}$ 且 $u_{i, l}<s_{j, u}<u_{i, u}+\operatorname{maxSRS}$.

证明 由 (1) 得 $s_{j, l}<u_{i, u}$, 由 (2) 得 $s_{j, u}>u_{i, l}, s_{j, u}-s_{j, l}>u_{i, l}-s_{j, l}, s_{j, l}>u_{i, l}-\left(s_{j, u}-s_{j, l}\right)$, 由 定义 1 得 $s_{j, l}>u_{i, l}-\operatorname{maxSRS}$, 由此得证 $u_{i, l}-\operatorname{maxSRS}<s_{j, l}<u_{i, u}$.

类似, 我们可以证明 $u_{i, l}<s_{i, u}<u_{i, u}+\operatorname{maxSRS}$.

定理 2 对任意 $u_{j} \in \psi_{i}^{d}$, 有 $s_{i, l}-\operatorname{maxURS}<u_{j, l}<s_{i, u}$ 且 $s_{i, l}<u_{j, u}<s_{i, u}+\max \mathrm{URS}$.

定理 2 的证明与定理 1 类似.

如前文所述, 场景中实体的频繁移动会相应带来实体区域的变化. 为研究区域变化对区域匹配关 系的影响, 我们以一个简单场景为例. 图 3 给出了 $X$ 轴上的一个更新区域 $u$ 和 5 个订购区域, 图中 $u^{\prime}$ 和 $u^{\prime \prime}$ 是更新区域 $u$ 的两个变化实例.

我们可以把限域的改变看做是限域两个端点移动之后的结果. 如图 3 所示, 当 $u$ 的下端点 $u_{l}$ 经 过 $s_{1}$ 的上端点 $s_{1 u}$ 向下移动到 $u_{l}^{\prime}, u$ 和 $s_{1}$ 出现交叠; 类似地, 当 $u_{u}$ 经过 $s_{3 l}$ 向下移动到 $u_{u}^{\prime}$ 时, $u$ 和 $s_{3}$ 不再交叠; 当 $u_{l}$ 经过 $s_{2 u}$ 和 $s_{3 u}$ 向上移动到 $u_{l}^{\prime \prime}, u$ 和 $s_{2}, s_{3}$ 不再交叠; 当 $u_{u}$ 经过 $s_{4 l}$ 和 $s_{5 l}$ 向上移 动到 $u_{u}^{\prime \prime}$ 时, $u$ 和 $s_{4}, s_{5}$ 出现交叠.

由上我们得到 4 个重要推论. 给定一个更新区域 $u_{i}$ 和一个订购区域 $s_{j}$, 有如下推论:

推论 1 若 $s_{j, l}$ 经过 $u_{i, u}$ 向坐标轴负方向移动, 则 $s_{j}$ 和 $u_{i}$ 出现交叠.

推论 2 若 $s_{j, l}$ 经过 $u_{i, u}$ 向坐标轴正方向移动, 则 $s_{j}$ 和 $u_{i}$ 不再交叠.

推论 3 若 $s_{j, u}$ 经过 $u_{i, l}$ 向坐标轴负方向移动, 则 $s_{j}$ 和 $u_{i}$ 不再交叠.

推论 4 若 $s_{j, u}$ 经过 $u_{i, l}$ 向坐标轴正方向移动, 则 $s_{j}$ 和 $u_{i}$ 出现交叠.

本文提出的区域匹配算法就是在以上推论基础上给出的.

\section{4 算法描述}

本节介绍我们提出的 DRBM 如何快速计算区域匹配信息和如何高效传输区域信息, 减少网络带 宽占用. 


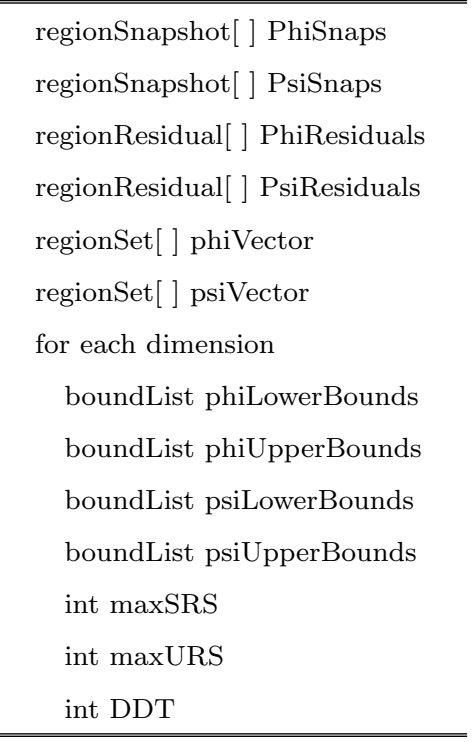

图 4 数据结构

Figure 4 Data structure

\section{1 数据结构}

本文算法所采用的数据结构如图 4 所示. 数组 PsiSnaps 存储所有更新区域的快照信息, 数组 PhiSnaps 存储所有订购区域的快照信息. 数组 PsiResiduals 存储所有更新区域的残差信息, 数组 PhiResiduals 存储所有订购区域的残差信息 (快照与残差详见 4.3 小节). 数组 phiVector 和 psiVector 分别存 储区域 $u_{i}$ 和 $s_{j}$ 的匹配区域信息 $\phi_{i}$ 和 $\psi_{j}$. 在每一维上分别有 4 个升序排序的端点列表和两个整形数 据. 列表 phiLowerBounds 存储所有订购限域的下端点, 列表 phiUpperBounds 存储所有订购限域的上 端点; 列表 psiLowerBounds 存储所有更新限域的下端点, 列表 psiUpperBounds 存储所有更新限域的 上端点; maxURS 和 maxSRS 分别记录最大更新限域长度和最大订购限域长度. DDT 表示维上最大 变化阈值 (详见 4.3 小节).

\section{2 匹配计算}

订购区域和更新区域的匹配信息计算过程相同, 因此, 为我们以更新区域的匹配信息计算为例来 介绍本文算法的匹配过程.

根据区域变化对区域间匹配关系的影响, 我们将区域的变化划分为两种类型: 创建新区域、改变 已有区域. 当新建一个区域, 算法通过匹配过程计算相关区域的匹配信息; 当改变一个现有区域, 算法 通过重匹配过程更新相关区域的匹配信息.

图 5 给出了二维共享空间中的一个简单场景, 场景中共有一个更新区域 $u_{i}$ 和 5 个订购区域. 算 法的目标就是利用最少的比较次数得到更新区域 $u_{i}$ 的准确匹配信息.

由于匹配过程是按维进行的, 为简化匹配查找过程, 首先采用排序的思想将每一维上所有订购区 域的上端点和下端点分别按升序排列在两个列表中. 因此, 在进行匹配查找之前先将每一维上所有订 购区域的上端点和下端点分别进行排序 [11]. 在图 5 所示场景中, 得到 4 个端点列表.

1) $X$ 轴下端点列表: $x$-phiLowerBounds $=\left\{s_{1 l}, s_{2 l}, s_{4 l}, s_{5 l}, s_{3 l}\right\}$; 

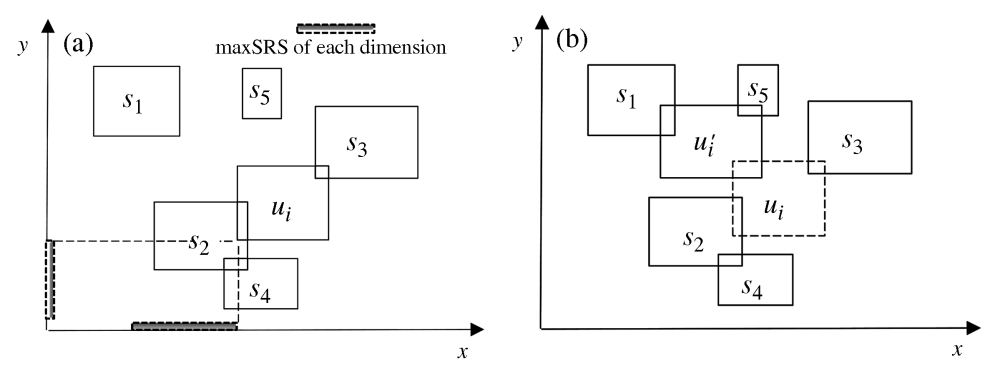

图 5 区域变化实例

Figure 5 Basic scenario for the DRBM algorithm. (a) Matching triggered by creating a new region; (b) rematching triggered by region modification

2) $X$ 轴上端点列表: $x$-phiUpperBounds $=\left\{s_{1 u}, s_{2 u}, s_{5 u}, s_{4 u}, s_{3 u}\right\}$;

3) $Y$ 轴下端点列表: $y$-phiLowerBounds $=\left\{s_{4 l}, s_{2 l}, s_{3 l}, s_{1 l}, s_{5 l}\right\}$;

4) $Y$ 轴上端点列表: $y$-phiUpperBounds $=\left\{s_{4 u}, s_{2 u}, s_{3 u}, s_{5 u}, s_{1 u}\right\}$.

将区域顶点排序之后, DRBM 算法分别按照以下方式处理两种类型的区域变化.

1) 创建区域. 当创建一个新的区域, DRBM 算法按照以下步骤进行匹配扫描.

步骤 1. 在每一维上, 根据定理 1 扫描下端点列表 phiLowerBounds, 查找所有下端点在区间 $\left(u_{i, l}\right.$ $\left.\operatorname{maxSRS}, u_{i, u}\right)$ 的订购区域.

以图 5(a) 所示场景为例, 当创建一个更新区域 $u_{i}$, 我们扫描得到两个区域集合: i) $X$ 轴上的区域 集合 $\operatorname{tempSet}_{x}=\left\{s_{2}, s_{4}, s_{5}, s_{3}\right\}$, ii) $Y$ 轴上的区域集合 $\operatorname{tempSet}_{y}=\left\{s_{4}, s_{2}, s_{3}\right\}$.

步骤 2. 对于步骤 1 的所有集合, 查找并去掉其中上端点小于 $u_{i, l}$ 的区域.

在图 5(a) 所示场景中, 得到两个集合: i) $X$ 轴上的区域集合 $\phi_{i}^{x}=\left\{s_{4}, s_{2}, s_{5}, s_{3}\right\}$, ii) $Y$ 轴上的区 域集合 $\phi_{i}^{y}=\left\{s_{2}, s_{3}\right\}$. 由不等式 (1), (2) 和定理 1 可知, $\phi_{i}^{x}$ 和 $\phi_{i}^{y}$ 中的区域分别在各自坐标轴上与更新 区域 $u_{i}$ 相交叠.

步骤 3. 按照公式 (3) 计算步骤 2 所得所有集合的交集, 得到准确地与 $u_{i}$ 相交叠的区域集合 $\phi_{i}$.

$$
\phi_{i}=\bigcup_{d} \phi_{i}^{d}
$$

于是, 对于图 5(a) 中场景, $\phi_{i}=\phi_{i}^{x} \cap \phi_{i}^{y}=\left\{s_{4}, s_{2}, s_{5}, s_{3}\right\} \cap\left\{s_{2}, s_{3}\right\}=\left\{s_{2}, s_{3}\right\}$.

2) 改变已有区域. 如果更新区域 $u_{i}$ 改变, DRBM 算法通过重匹配过程更新匹配信息. 重匹配的过 程包括以下步骤.

步骤 1 . 在每一维上, 扫描上端点列表 phiUpperBounds, 查找所有上端点在 $u_{i}$ 下端点移动范围内 的区域; 扫描下端点列表 phiLowerBounds, 查找所有下端点在 $u_{i}$ 上端点移动范围内的区域.

以图 5(b) 所示场景为例, 当区域 $u_{i}$ 移动到 $u_{i}^{\prime}$ 时, 我们得到 4 个区域集合: $\operatorname{tempSet}_{x, l}, \operatorname{tempSet}_{x, u}$, tempSet $y, l$ 和 tempSet $_{y, u}$. 其中, 通过在 $x$-phiLowerBounds 上扫描端点范围在 $\left(u_{i, u}^{\prime}, u_{i, u}\right)$ 的区域得 到 tempSet ${ }_{x, l}=\left\{s_{3}\right\}$; 在 $x$-phiUpperBounds 上扫描端点范围在 $\left(u_{i, l}^{\prime}, u_{i, l}\right)$ 的区域得到 $\operatorname{tempSet}_{x, u}$ $=\left\{s_{1}\right\}$; 在 $y$-phiLowerBounds 上扫描端点范围在 $\left(u_{i, u}, u_{i, u}^{\prime}\right)$ 的区域得到 $\operatorname{tempSet}_{y, l}=\left\{s_{1}, s_{5}\right\}$; 在 $y$-phiUpperBounds 上扫描端点范围在 $\left(u_{i, l}, u_{i, l}^{\prime}\right)$ 的区域得到 $\operatorname{tempSet}_{y, u}=\left\{s_{2}\right\}$.

步骤 2 . 在每一维上, 根据步骤 1 的计算结果得到两个区域集合: i) 与区域 $u_{i}$ 新出现交叠的区域 集合 $\phi_{i}^{d+}$, ii) 与区域 $u_{i}$ 不再交叠的区域集合 $\phi_{i}^{d-}$. 
集合 $\phi_{i}^{d+}$ 和 $\phi_{i}^{d-}$ 都是根据第 3 节中的推论进行计算得到的. 以图 5(b) 为例, 当 $u_{i, l}$ 沿 $X$ 轴负方 向移动, 由推论 1 得 $\operatorname{tempSet}_{x, u} \in \phi_{i}^{d+}$; 当 $u_{i, u}$ 沿 $X$ 轴负方向移动, 由推论 3 得: $\operatorname{tempSet}_{x, l} \in \phi_{i}^{d-}$; 同 理可得 tempSet ${ }_{y, u} \in \phi_{i}^{d-}$, 以及 $\operatorname{tempSet}_{y, l} \in \phi_{i}^{d+}$. 至此我们可以得到 $\phi_{i}^{x+}=\emptyset \cup \operatorname{tempSet}_{x, u}=\emptyset \cup\left\{s_{1}\right\}$ $=\left\{s_{1}\right\} ; \phi_{i}^{x-}=\emptyset \cup \operatorname{tempSet}_{x, l}=\emptyset \cup\left\{s_{3}\right\}=\left\{s_{3}\right\} ; \phi_{i}^{y+}=\emptyset \cup \operatorname{tempSet}_{y, l}=\emptyset \cup\left\{s_{1}\right\}=\left\{s_{1}, s_{5}\right\} ; \phi_{i}^{y-}=$ $\emptyset \cup$ tempSet $_{y, u}=\emptyset \cup\left\{s_{2}\right\}=\left\{s_{2}\right\}$.

步骤 3. 计算 $\phi_{i}^{+}=\bigcup_{d} \phi_{i}^{d+}$ 和 $\phi_{i}^{-}=\bigcup_{d} \phi_{i}^{d-}$. 然后扫描所有维中不满足不等式 (1) 和 (1) 的区域, 并从集合 $\phi_{i}^{+}$中去除.

于是, 在图 5(b) 中我们得 $\phi_{i}^{+}=\left\{s_{1}, s_{5}\right\}$ 和 $\phi_{i}^{-}=\left\{s_{2}, s_{3}\right\}$.

值得注意的是, 当在任意维度上出现 $u_{i, l}^{\prime}$ 大于 $u_{i, u}+\operatorname{maxSRS}$ 或者 $u_{i, u}^{\prime}$ 小于 $u_{i, l}-\operatorname{maxSRS}$, 根据 定理 1,2 可知, 所有之前与区域 $u_{i}$ 的匹配都消失, 也就是 $\varphi_{i}^{-}=\varphi_{i}$. 这时, 可以像创建新区域一样, 在 每一维上查找 $\left(u_{i, l}^{\prime}-\operatorname{maxSRS}, u_{i, u}^{\prime}\right)$ 范围内同时满足不等式 (1), (2) 的区域来得到集合 $\varphi_{i}^{d+}$, 从而代替 步骤 1 和 2 , 提高匹配效率.

步骤 4 . 根据式 (4) 更新 $\phi_{i}$.

$$
\phi_{i}=\phi_{i} \cup \phi_{i}^{+}-\phi_{i}^{-} .
$$

图 5(b) 中我们可得 $\phi_{i}=\phi_{i} \cup \phi_{i}^{+}-\phi_{i}^{-}=\left\{s_{2}, s_{3}\right\} \cup\left\{s_{1}, s_{5}\right\}-\left\{s_{2}, s_{3}\right\}=\left\{s_{1}, s_{5}\right\}$.

表 1 利用我们上面定义的符号给出了 DRBM 匹配方法的步骤. 算法对于订购区域匹配信息的计 算具有相同的步骤.

\section{3 区域传输}

区域信息的传输在 DDM 服务应用中非常重要, 例如当只有区域的一个限域发生改变的时候, 传 输所有区域数据将明显带来网络带宽的额外消耗. 为避免这种区域传输造成的不必要的带宽浪费, 本 文引入了区域快照与区域残差的概念. 区域快照是指某一时刻区域信息的一个完整备份. 在任意仿真 时刻下, 整个仿真环境只能存在某一区域的单一快照. 如果区域快照被更新, 区域快照版本也需要被 相应更新.

为方便描述快照和残差策略, 我们首先举一个简单例子进行说明. 图 6 给出了区域 $R$ 随仿真推 进的变化过程, 为描述方便我们只让 $R$ 在 $X$ 轴上变化. 如图 6(a) 所示, 区域 $R$ 在 $t_{0}$ 时刻创建了 一份快照. 当区域 $R$ 改变时, 通过计算所有维上区域的限域相对区域当前快照的位移量得到一份残 差信息. 以图 6 为例, 当区域的限域 $r_{x}$ 在 $t_{1}$ 时刻改变后, 我们得到残差 $r_{x}(a-c, b-d)$. 因此, 只需 要传输残差 $r_{x}(a-c, b-d)$ 和相应的快照版本来完整的区域信息, 从而减少网络带宽占用. 接收方根 据接收到的残差信息更新相应的端点列表 (即 phiLowerBounds, phiUpperBounds, psiLowerBounds 或 psiUpperBounds). 因此, 本文方法的区域更新报文就分为区域快照更新报文和一系列的相对于当前区 域快照的小数据量区域残差报文.

另外, 在快照残差策略下, 我们可以很容易通过一次将所有残差信息打包、压缩然后一次性发送 来进一步减少网络带宽. 值得一提的是, 区域残差值越小, 数据的压缩比越高, 因为残差越小高位的 零值数据越多. 因此, 为使残差数据保持在较小范围内, 我们引入维上最大变化阈值 $(\delta$, dimension displacement threshold). 路由空间中的每一维度都有自己的维上最大变化阈值 $\delta$, 当区域变化在任一 维度上有超过阈值 $\delta$, 就为该区域创建一个新版本的快照. 以图 6 为例, $t_{n}$ 时刻 $r_{x}$ 的上端点位移长度 $|b-f|$, 超过 $X$ 轴上的阈值 $\delta$, 此时 $R$ 的新版本的快照产生. 
表 1 DRBM 匹配算法

Table 1 The matching algorithm

\begin{tabular}{|c|c|}
\hline 01. if (create a new region) & 19. if(modify an existing region) \\
\hline 02. if (an update region $U_{i}$ is created) & 20. if (an subscription region $S_{i}$ is modified) \\
\hline for each dimension $d$ & for each modified range $s_{i}$ \\
\hline insert $u_{i, l}$ into the psiLowerBounds & $/ / s_{i}$ is in dimension $d$ \\
\hline insert $u_{i, u}$ into the psiUpperBounds & resort the phiLowerBounds of $d$ \\
\hline if (size of $\left.u_{i}>\operatorname{maxURS}\right)$ & resort the phiUpperBounds of $d$ \\
\hline $\max U R S=$ size of $u_{i}$ & if (size of $s_{i}>$ maxSRS) \\
\hline end & $\operatorname{maxSRS}=$ size of $s_{i}$ \\
\hline compute $\phi_{i}^{d}$ & end \\
\hline end & compute $\psi_{i}^{d+}$ and $\psi_{i}^{d-}$ \\
\hline compute $\phi_{i}$ as in Eq. (3) & end//end of for \\
\hline for each subscription region $S_{j} \in \phi_{i}$ & compute $\psi_{i}^{+}$and $\psi_{i}^{-}$ \\
\hline add $U_{i}$ to $\psi_{j}$ & update $\psi_{i}$ as in Eq. (4) \\
\hline 14. end & for each update region $U_{j} \in \psi_{i}^{+}$ \\
\hline 15. else //a subscription region is created & add $S_{i}$ to $\phi_{j}$ \\
\hline similar to above ... & end \\
\hline 17. end & for each update region $U_{k} \in \psi_{i}^{-}$ \\
\hline 18. end & remove $S_{i}$ from $\phi_{k}$ \\
\hline & end \\
\hline & 38. else //a update region is modified \\
\hline & similar to above . \\
\hline & 40. end \\
\hline & 41. end \\
\hline
\end{tabular}

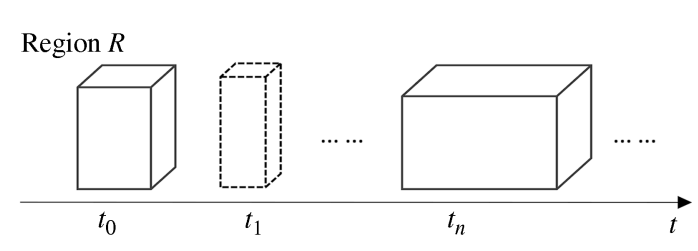

(a)

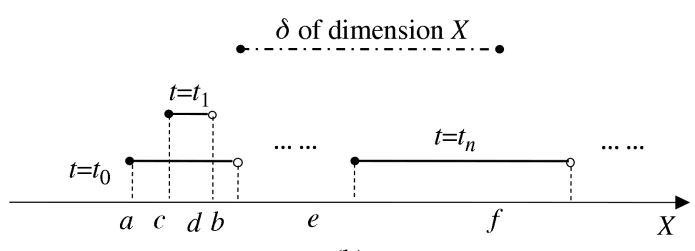

(b)

图 6 区域快照及残差

Figure 6 Snapshot and its residual of region $R$ as the simulation time proceeds. (a) Region $R$ changes as simulation time goes on; (b) range changes accordingly in dimension $X$

\section{5 算法效率理论分析}

为深入分析本文算法的性能, 本节给出算法在空间和时间上的复杂度分析. 首先给出复杂度分析 所用到的一些主要参数. 
- 发布区域数量 $N$.

- 订购区域数量 $N$ (为分析和表述简单, 我们假设订购区域与发布区域数量相同).

・路由空间维度 $D$.

- 每个维度的区间范围 $[0, L]$.

- 每一维上的最大限域长度 maxRS.

为分析算法的空间复杂度, 我们再对图 4 进行分析. 图 4 中, 区域快照数组 PsiSnaps 和 PhiSnaps 占用 $\mathrm{O}(N)$ 存储空间, 残差数组 PsiResiduals 和 PsiResiduals 空间复杂度也是 $\mathrm{O}(N)$. 根据文献 [10], 对 于一个给定区域, 与其匹配的区域平均数目为 $\mathrm{O}\left((\operatorname{maxRS} / L)^{D *} N\right)$. 因此, 数组 phiVector 和 psiVector 需占用 $\mathrm{O}\left((\operatorname{maxRS} / L)^{D *} N^{2}\right)$ 存储空间. 区域端点列表需要 $\mathrm{O}(N)$ 存储空间, maxSRS 和 maxURS 存 储空间为 $O(1)$. 总体来看, 本文 DRBM 算法的存储复杂度为 $O\left((\operatorname{maxRS} / L)^{D *} N^{2}\right)$. 尽管复杂度仍然 是 $N$ 的平方级, 但是算法实际存储空间需求取决于数值 $(\operatorname{maxRS} / L)^{D}$ 的大小, $(\operatorname{maxRS} / L)^{D}$ 在大的路 由空间中往往是非常小的, 例如 $0.001^{2}$.

为分析算法的时间复杂度, 我们再对表 1 进行分析. 表 1 中将顶点插入相应端点列表以及计算最大 限域长度的过程 (步骤 4 8) 的计算复杂度为 $O(\log N+1)$; 步骤 9 计算 $\phi_{i}^{d}$ 的复杂度为 $O\left(\operatorname{maxRS} / L^{*} N\right)$; 步骤 11 需要 $O\left(\operatorname{maxRS} / L^{*} N\right)$ 次计算; 步骤 12 14 的计算复杂度与单个区域平均匹配数目成正比, 平 均计算次数为 $O\left((\operatorname{maxRS} / L)^{D *} N\right)$; 当某个现有区域被改变, 对端点列表进行重排序以及计算最大限 域长度的过程 (步骤 23 27) 计算复杂度为 $O(\log N+1)$; 步骤 28 计算复杂度为 $O\left(\operatorname{maxRS} / L^{*} N\right)$; 步骤 $30 \sim 37$ 的计算量与 $\phi_{i}^{d+}$ 和 $\phi_{i}^{d-}$ 的大小成正比, 其计算复杂度为 $O\left(\operatorname{maxRS} / L^{*} N\right)$.

总体来看, 本文算法在创建新区域和改变已有区域时候的匹配计算复杂度为 $O\left(\operatorname{maxRS} / L^{*} N\right)$. 然 而 maxRS/ $L$ 的数值在大场景中通常很小, 例如 0.001 , 因此本文提出的 DRBM 算法应该具有很好的 效率.

\section{6 实验}

本节对新算法的效率进行了验证, 包括匹配计算的效率、区域传输的带宽占用以及 DDM 服务的 响应时延.

为验证上一节中对算法进行的理论分析, 我们采用 $\mathrm{C}++$ 分别实现了基于区域的匹配算法、改进 的基于排序的匹配算法, 以及本文提出的 DRBM 算法, 并对 3 种算法进行测试. 每个测试过程都被重 复执行 50 次并取其平均值. 所有的程序都是在同一台 $\mathrm{PC}$ 上运行的, 配置为奔腾 D3.40 GHz CPU、 1 GB 内存, 操作系统为 Microsoft Windows XP.

为比较以上算法在不同区域规模下的匹配效率, 我们设计了两组实验. 实验中, 取 $L=1000000$, 取 $\operatorname{maxRS}=100$. 把大约 $80 \%$ 的区域位移限制在 $\operatorname{maxRS} \times 2$ 以下, 另外的部分限制在区间 $[\operatorname{maxRS} \times 2$, $\operatorname{maxRS} \times 10]$ 内.

图 7 给出了一个区域改变情况下, 不同算法在不同区域数量规模下进行匹配计算所消耗的时间. 从图 7(a) 可以看出, 与其他两种算法相比, 随着区域数目的增加, 基于区域的匹配算法所消耗的匹配 时间快速增加. 图 7(b) 给出了改进的排序算法与本文算法的清晰对比, 可以看出, 本文算法在区域数 量逐渐增加的情况下, 具有更高的匹配效率.

图 8 给出了在更大规模区域数目下区域改变后算法匹配计算所消耗的时间. 可以看出, 在区域规 模逐渐增大的情况下, 本文算法比改进的排序算法明显具有更高的匹配计算效率. 

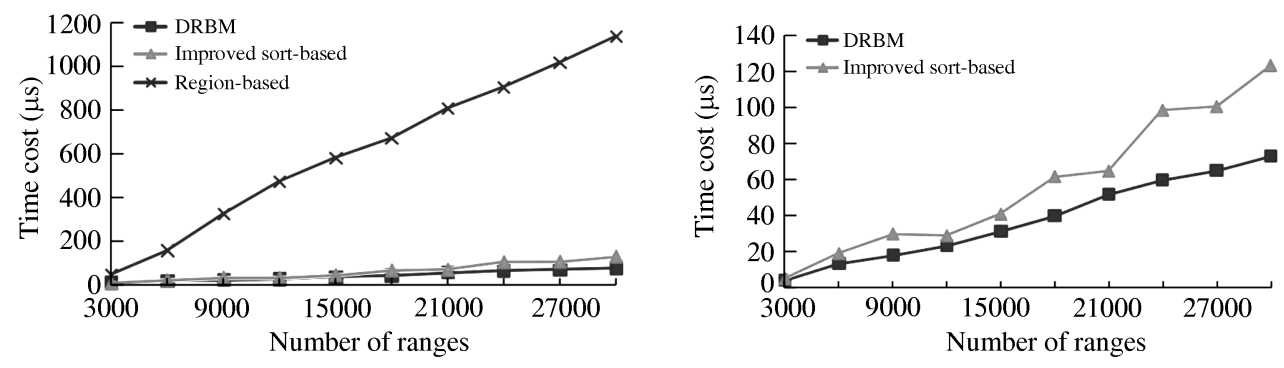

图 7 区域数量对匹配效率的影响

Figure 7 Performance with respect to number of ranges

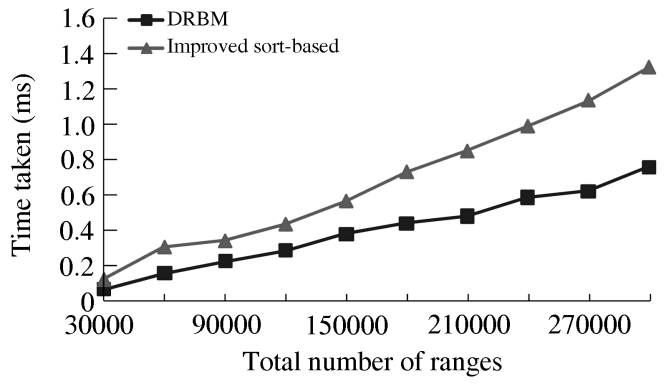

图 8 大量区域对匹配效率的影响

Figure 8 Performance with respect to a large number of ranges

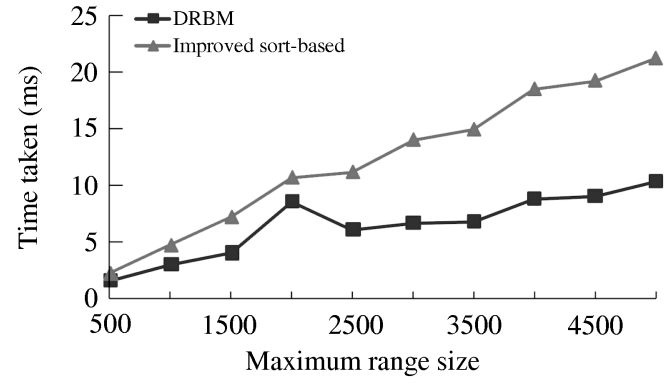

图 9 区域大小对匹配效率的影响

Figure 9 Performance with respect to maximum range size

由于基于区域的匹配算法复杂度仅与区域的数目有关, 我们对本文 DRBM 算法和基于排序的匹 配算法在不同区域大小情况下的匹配效率进行了对比实验. 我们让最大限域长度从 500 到 5000 按照 500 的步长进行实验, 结果如图 9 所示. 从图 9 中可以看出, 随着 $\operatorname{maxRS} / L^{*} N$ 不断增加, 本文算法明 显比基于排序的算法具有更高的效率.

在数据分发管理服务中, 匹配算法所占用的存储空间大小十分重要, 尤其是在大规模的仿真环境 中. 因此, 本文对大区域数量下算法的存储空间占用量进行了测试. 图 10 给出了测试的结果, 可以看 出, 本文 DRBM 算法和基于排序的算法因为建立了额外的排序索引, 比基于区域的算法占用了更多的 内存空间. 本文 DRBM 算法由于需要建立快照信息, 占用了更多的内存. 好的方面是, DRBM 算法在 $N=300000$ 的大规模场景中占用的内存空间不超过 $120 \mathrm{MB}$, 这在硬件不断升级的今天是可以接受的.

我们已将本文 DRBM 算法实现到 BH RTI 2.3 中. 为验证本文的区域传输策略占用更少的网络 带宽, 我们设计和实现了一种简单的 HLA 盟员程序, 并应用到 BH RTI 2.3 和另外两个国际主流 RTI 软件 DMSO RTI 1.3NGv6 (non-bundle) 和 MÄK RTI 3.0 进行对比实验, 实验中的盟员数量从 2 逐渐 增加到 8. 因为需要测试的是区域传输所占用的网络带宽, 我们让每个盟员创建 500 个区域, 并且只 触发 RTI 软件的 DDM 服务. 所有的盟员都在单独的机器上运行, 并且所有的机器都连接在同一局域 网内.

图 11 给出了利用 PerfCounter 测量出来的网络带宽, 结果表明相比 DMSO RTI 和 MÄK RTI, BH RTI 节约了大约 $15 \%$ 的网络带宽.

数据分发管理服务响应延迟是指一个 DDM 服务接口被调用, 经过多长时间这个调用产生的结果 到达受这个调用影响的仿真盟员. 在本次实验中, 我们分两组测试了 DDM 服务接口 registerObjectIn- 


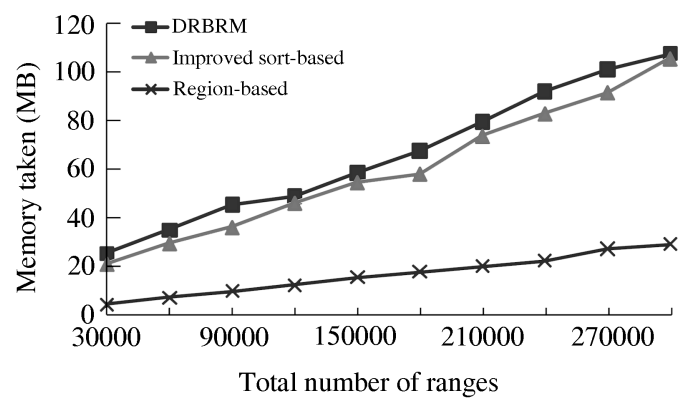

图 10 内存占用

Figure 10 Memory taken

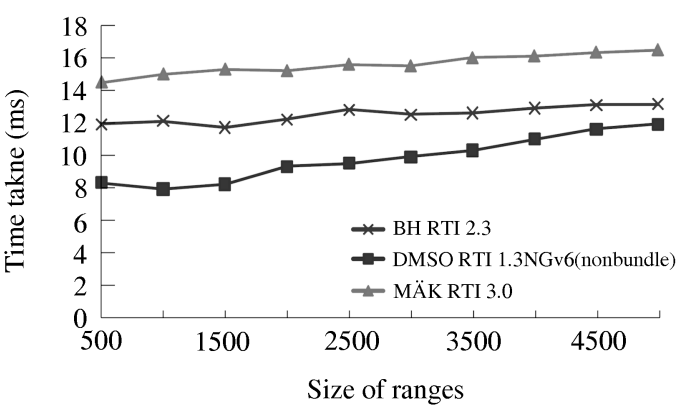

图 12 区域大小对 DDM 响应延迟的影响

Figure 12 DDM services response delay with respect to range size

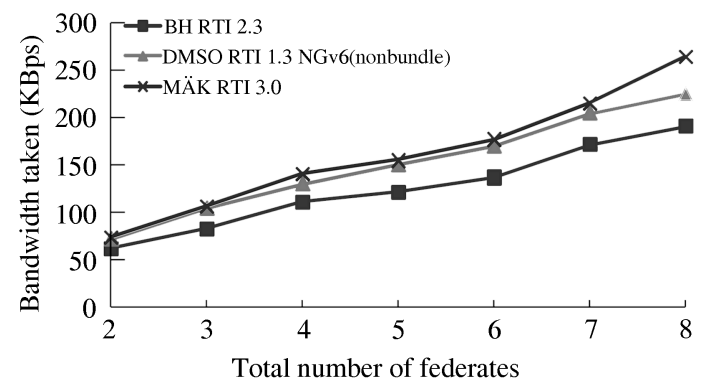

图 11 带宽占用

Figure 11 Bandwidth cost

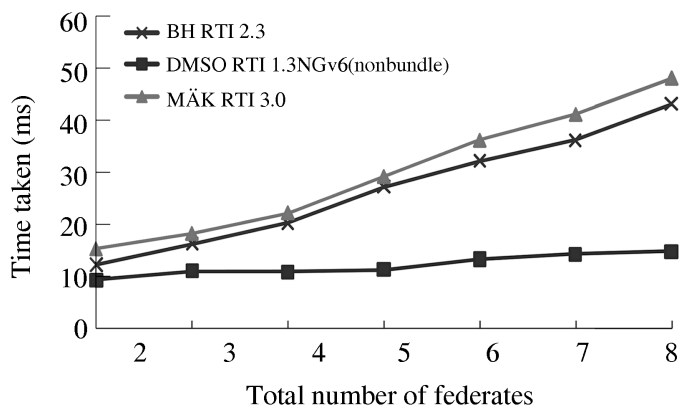

图 13 区域数量对 DDM 响应延迟的影响

Figure 13 services response delay with respect to the number of rederates

stanceWithRegion 所消耗的时间. 由于测试在局域网中进行, 网络时延相对于处理时间来说相对较小, 所以这里忽略掉网络延迟.

第一组实验测量区域大小对数据分发管理服务响应延迟的影响. 测试共有 3 个盟员, 每个盟员创 建大小相同的 510 个区域, 其中 500 个更新区域, 10 个订购区域. 测试结果如图 12 所示, 所有 RTI 软 件都有较稳定的响应时延. 其中 DMSO RTI 由于采用效率较低的基于区域的匹配算法, 时延相对较 长.

第二组实验测试区域数量对 DDM 服务响应延迟的影响. 与第一组测试不同的是, 本组测试将区 域大小固定为 500 , 仿真盟员数量从 2 增加到 8 , 因此区域数量也相应增加. 从图 13 的测试结果中可 以看出, 随着区域数量的增加, BH RTI 明显比 DMSO RTI 和 MÄK RTI 拥有更快的响应速度. 主要 原因是后两者都采用单服务器结构, 随着区域数目的增加服务器负载也随之快速增加. 而 BH RTI 采 用多服务器结构, 计算负载被有效分散, 从而提高了服务响应速度.

\section{7 结论}

本文提出了一种基于位移残差的数据分发管理服务算法 DRBM. 该算法能够有效减少区域变化 所带来的匹配关系比较次数, 在具有较高区域匹配计算效率的同时, 能够有效减少区域传输所消耗的 网络带宽. 
与之前算法不同的是, 本文算法兼顾了兴趣区域的匹配效率和区域传输的效率. 首先定义了区域 变化与区域匹配变化之间的映射关系, 采用该映射可以明显减少区域改变后所需要的匹配计算次数, 从而提高了匹配效率; 将区域更新信息划分为两种数据表达, 区域快照与区域残差. 通过尽量传输区 域残差来代替传输完整的区域信息可以有效减少区域信息传输所消耗的网络带宽. 在此基础上, 本文 提出一种基于位移残差的 DDM 匹配与传输算法 DRBM. 实验结果表明, DRBM 算法具有良好的匹配 效率, 并且能够大大减少网络带宽占用, 尤其是在具有大量不断变化区域的情况下.

\title{
参考文献
}

1 U.S. Department of Defense, High Level Architecture (HLA)-Federate Interface Specification, Version 1.3, 1998

2 Van Hook D J, Calvin J O. Data distribution management in RTI 1.3. In: Proceedings of the 1998 Spring Simulation Interoperability Workshop, Orlando, 1998. 9-13

3 Ayani R, Moradi F, Tan G. Optimizing cell size in grid-based DDM. In: Proceedings of the 14th Workshop on Parallel and Distributed Simulation, Bologna, 2000. 93-100

4 Rak S J, Van Hook D J. Evaluation of grid-based relevance filtering for multicast group assignment. In: Proceedings of the Distributed Interactive Simulation, Orlando, 1996. 739-747

5 Lo S H, Chiu C A, Pai F P, et al. MGRID: a modifiable-grid region matching approach for DDM in the HLA RTI. In: Proceedings of the 2009 Spring Simulation Multiconference, San Diego, 2009. 114

6 Abrams H, Watson K, Zyda M. Three-tiered interest management for large-scale virtual environments. In: Proceedings of the ACM Symposium on Virtual Reality Software and Technology, Taiwan, 1998. 125-129

7 Tan G, Zhang Y, Ayani R. A hybrid approach to data distribution management. In: Proceedings of the 4th IEEE International Workshop on Distributed Simulation and Real-Time Applications, San Francisco, 2000. 55-61

8 Zhang G, Zhang X, Li D. A hybrid DDM algorithm based on weight function. In: 5th International Conference on Fuzzy Systems and Knowledge Discovery, Jinan, 2008. 225-229

9 Pan K, Turner S, Cai W, et al. Implementation of data distribution management services in a service oriented HLA RTI. In: Proceedings of the 2009 Winter Simulation Conference, Austin, 2009. 1027-1038

10 Pan K, Turner S, Cai W, et al. An efficient sort-based DDM matching algorithm for HLA applications with a large spatial environment. In: 21st International Workshop on Principles of Advanced and Distributed Simulation (PADS 07), Washington, 2007. 70-82

11 Raczy C, Tan G, Yu J. A sort-based DMM matching algorithm for HLA. ACM Trans Model Comput Simul, 2005, 15: $14-38$

12 Capps M, Stotts D. Research issues in developing networked virtual realities. IEEE Comput Soc, 1997, 18: 205-211

13 Macedonia M, Zyda M, Pratt D, et al. Exploiting reality with multicast groups: A network architecture for large-scale virtual environments. IEEE Comput Graph Appl, 1995, 15: 38-45

\section{Displacement residual based DDM matching algorithm}

\author{
ZHANG $\operatorname{Lin}^{1,2 *}$, ZHOU Zhong ${ }^{1,2}$, LIU $\operatorname{Lin}^{1,2} \&$ WU Wei ${ }^{1,2}$ \\ 1 State Key Laboratory of Virtual Reality Technology and Systems, Beihang University, Beijing 100191, China; \\ 2 School of Computer Science and Engineering, Beihang University, Beijing 100191, China \\ *E-mail: zhanglin@vrlab.buaa.edu.cn
}

\begin{abstract}
High level architecture (HLA) is the prevailing standard for modeling and simulation. The data distribution management (DDM) service of HLA is defined for reducing the delivery of irrelevant data. The key
\end{abstract}


in DDM implementation is the region overlap computation, i.e. the matching between update and subscription regions. Existing algorithms usually make a compromise between region fidelity and network payload. This paper takes both the matching algorithm efficiency and bandwidth cost into account. The main contributions are: 1) illustrating the relationship between region changes and overlap changes, as helps reduce the number of region matching and then improves the total matching efficiency; 2) classifying region updates into two types of data expression, snapshot and residual. The network traffic will be reduced by transmitting only residual data instead of full region representations occasionally. Consequently, a region matching algorithm called displacement residualbased DDM matching (DRBM) is proposed in the paper. Theoretical analysis, algorithm implementation and experiment evaluation are presented. Experiment results show that DRBM provides better matching performance and significant network payload reductions especially when there is a large number of changing regions.

Keywords distributed simulation, high level architecture, data distribution management, region snapshot, region residual

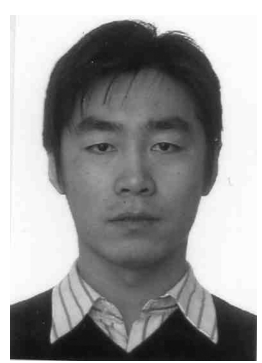

ZHANG Lin was born in 1982. $\mathrm{He}$ received the M.S. degree in computer software engineering from Ocean University of China, Qingdao in 2008 Currently, he is a Ph.D. student at Beihang University. His research interests include distributed simulation, virtual reality, tele-immersion, and telepresence.

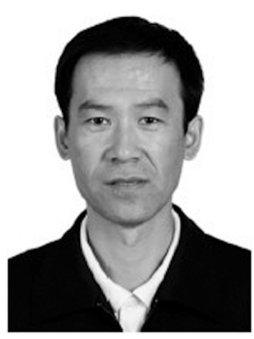

WU Wei is a Professor in School of Computer Science and Engineering at Beihang University, currently the chair of TCVRV (Technical Committee on Virtual Reality and Visualization) of CCF (China Computer Federation). He received the Ph.D. degree from Harbin Institute of Technology in 1995. He has published more than 90 papers, 33 issued patents and 1 book. His current research interests involve real-time $3 \mathrm{D}$ reconstruction, remote immersive system and augmented reality.

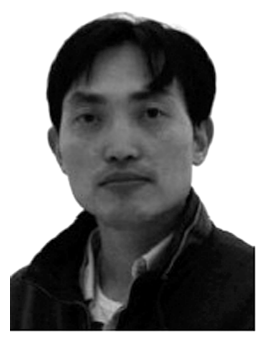

ZHOU Zhong is an Associate Professor at State Key Lab of Virtual Reality Technology and Systems, Beihang University. He received his B.S. degree from Nanjing University and Ph.D. degree from Beihang University in 1999 and 2005 respectively. His main research interests include tele-immersion, natural phenomena simulation, distributed virtual environment and Internet based VR technologies. He is member of $\mathrm{CCF}, \mathrm{ACM}$ and IEEE. 